\title{
La importancia de la encuesta de selección de donantes en el pretamizaje: experiencia en un banco de sangre de Bogotá, noviembre-diciembre de 1996
}

\author{
Mauricio Beltrán ${ }^{1}$, Maribel Ayala ${ }^{1}$, Jorge H. Jara ${ }^{2}$ \\ ${ }^{1}$ Grupo Banco de Sangre, Instituto Nacional de Salud, Bogotá, D.C., Colombia. \\ ${ }^{2}$ Centro Control de Enfermedades, Instituto Nacional de Salud, Bogotá, D.C., Colombia.
}

La disminución del riesgo de adquirir una enfermedad por vía transfusional se basa en la selección cuidadosa de los donantes por medio de la encuesta, el tamizaje serológico y, más recientemente, la inactivación viral. La encuesta permite detectar individuos en riesgo de portar infecciones que podrían no ser detectados en el tamizaje serológico (período de ventana); por tanto, su utilidad depende, entre otros, del tipo de donante y de sus motivaciones para donar. En algunos países, los donantes difieren de la población general en aspectos como las características sociales, la frecuencia en la donación y, principalmente, en el bajo riesgo de presentar infecciones transmisibles por sangre. En Colombia, sin embargo, cerca del $80 \%$ de la sangre proviene de donantes de reposición y muchas veces obligados, que donan con poca regularidad. Con el propósito de analizar la utilidad de la encuesta, el proceso de selección de donantes y estimar la prevalencia de las infecciones transmitidas por sangre tamizada, se realizó un estudio descriptivo de los registros de donantes que acudieron en noviembre y diciembre de 1996 a un banco de sangre de Bogotá. Se revisaron 414 encuestas de donantes y sus resultados serológicos correspondientes. Se analizó si la encuesta incluía o no preguntas sobre factores de riesgo para infecciones susceptibles de trasmitirse por sangre, el porcentaje de preguntas con respuesta en todos los casos, la concordancia entre las respuestas del donante y el procedimiento realizado, ya fuera aceptando o no la donación, las características sociodemográficas y, finalmente, se estimó la reactividad a anticuerpos contra VIH, VHC, Treponema pallidum, Trypanosoma cruzi y contra HBsAg.

Se encontró que las preguntas sobre antecedentes de tatuajes, uso de drogas intravenosas y tipo de conducta sexual, entre otras, no estaban incluidas en la encuesta. El $90 \%$ de las preguntas fue diligenciado en las 414 encuestas; las preguntas sobre sexo, ocupación y estado civil presentaron el mayor porcentaje sin respuesta. $65 \%$ de los donantes eran hombres. El promedio de edad fue de 32 años. Del total de donantes, $325(78,5 \%)$ no informaron ningún factor de riesgo para la donación en su encuesta y de éstos, $52(16 \%)$ fueron serorreactivos a alguno de los marcadores, mientras que, entre 89 donantes que informaron al menos un factor de riesgo, se hallaron 10 serorreactivos. La serorreactividad a todos los marcadores (VIH, HBsAg, VHC, T. pallidum y $T$. cruzi) fue de $15 \%$, tres veces mayor que el promedio nacional $(5,2 \%)$ en 1996 y al informado en otros estudios.

Los principales factores de riesgo informados por los donantes reactivos fueron: antecedente de transfusión, de radiografía de tórax y no gozar de buena salud en el momento de la donación. No hubo diferencias significativas estadisticamente en las características sociodemográficas para donantes reactivos y no reactivos. La encuesta busca seleccionar el donante con menos factores de riesgo, protegiendo así la salud del donante y del receptor de sangre y permitiendo, además, optimizar los recursos del banco.

Palabras clave: riesgo transfusional, encuesta, selección de donantes, banco de sangre. 


\title{
Importance of donor selection survey in infectious disease prescreening: experience at a blood bank in Bogota, November-December, 1996
}

\begin{abstract}
Decreasing the risk of acquiring a transfusional disease depends on a careful donor selection by means of surveys, serological screening and, more recently, viral inactivation. Surveys allow the detection of individuals who might be carriers of infections not detected by serological screening (window period), and, therefore, their usefulness depends, among others, on the type of donor and his/her motivation for doning. In some countries, donors differ from general population in aspects such as social characteristics, doning frequency and, above all, their low risk in blood transmitted infections. In Colombia, nevertheless, around $80 \%$ of the blood comes from replacement, and many times, forced donors who do it on a non regular basis. In order to assess the survey's usefulness and the donors' selection process, and to establish the prevalence of blood transmitted infections subject to screening, a descriptive study was carried out of donors' records who approached a blood bank in Bogotá during November and December, 1996. 414 donors' surveys and their corresponding serological results were reviewed. We assessed if the survey included or not questions related to risk factors for blood transmitted infections, the percentage of questions answered in each case, the relation between donors' answers and their acceptance or refusal to donning, sociodemographic characteristics, as well as reaction to antibodies against $\mathrm{HIV}, \mathrm{HCV}$, Treponema pallidum, Trypanosoma cruzi and HBsAg.

We found that questions regarding tattoos, use of intravenous drugs and types of sexual conduct, among others, were not included in the survey. $90 \%$ of the questions were answered in the 414 surveys, but questions on sex, occupation and marital status appeared more frequently unanswered. $65 \%$ of donors were men and the average age was 32 . From the total number of donors, $325(78.5 \%)$ did not report any risk factor for blood donation, and out of these, $52(16 \%)$ were seroreactive to some of the markers, while among the 89 donors who reported some risk factor, we found 10 seroreactive. $15 \%$ of seroreactivity to all markers such as $\mathrm{HIV}, \mathrm{HBsAg}, \mathrm{HCV}$, T. pallidum and T. cruzi was three times higher than the national average $(5,2 \%)$ in 1996 and to that reported in other studies. The main risk factors reported by reactive donors were: previous transfusions, chest film and poor health at the moment of donation. There were no statistically significant differences in sociodemographic characteristics between reactive and non reactive donors. The survey aims at selecting donors with the least risk factors, thus protecting both donors and recipients' health, and using blood bank's resources at their best.
\end{abstract}

Key words: transfusional risk, survey, donors selection, blood bank.

La seguridad de la sangre que se va a transfundir depende, principalmente, de la selección cuidadosa de los donantes por medio de la encuesta, el tamizaje serológico y, más recientemente, la inactivación viral y remoción de parásitos, hongos y bacterias. La encuesta previa al tamizaje permite detectar individuos con riesgo de portar infecciones que podrían no ser detectados en el tamizaje serológico (período de ventana) (1). La utilidad de la encuesta, por tanto, depende de un diseño correcto que involucre

\footnotetext{
Correspondencia:

Mauricio Beltrán, Instituto Nacional de Salud

bsangre@hemagogus.ins.gov.co

Teléfono 2220577 , extensiones 420 y 421

Fax: 222 0577, extensión 420
}

Recibido: 12/11/99; aceptado: 06/10/00 preguntas sobre los factores de riesgo, una adecuada aplicación (entrevista personal), información clara y concreta para el donante sobre la donación y sobre las infecciones transmitidas por sangre que despeje sus inquietudes, de manera que comprenda y responda con sinceridad, y de la adopción correcta de los criterios para aceptar o no al donante según sus respuestas (2).

Otros factores muy importantes de los cuales depende su utilidad, se basan en la población seleccionada y sus motivaciones para donar. En la mayoría de los países, los donantes de sangre difieren de la población general en aspectos como características sociodemográficas, estrato socioeconómico, altruismo para donar, frecuencia en la donación y, principalmente, en su bajo riesgo 
de presentar infecciones transmisibles por sangre. En Colombia, gran parte de la sangre que se recolecta $(80 \%)$, proviene de los bancos de sangre ubicados dentro de las instituciones hospitalarias, los cuales no realizan promoción de la donación y sus donantes son de reposición y, muchas veces, obligados a donar (3). Esto facilita que algunos donantes, por interés o presión, nieguen información relevante que los excluiría como donantes y, además, que donen con escasa frecuencia. Estos donantes provienen de población poco seleccionada que presenta mayor riesgo para la presencia de agentes infecciosos transmisibles por sangre (4).

En Colombia no se han hecho informes que permitan analizar el proceso de selección de donantes y la utilidad de la encuesta de selección. De igual manera, no se aplica a nivel nacional una encuesta unificada en los bancos de sangre, razón por la cual se justifica esta publicación. El objetivo de este trabajo fue analizar la utilidad de la encuesta, el proceso de selección de donantes de sangre y estimar la prevalencia para los distintos marcadores de infecciones tamizados en un banco de sangre.

\section{Materiales y métodos}

Se realizó un estudio descriptivo retrospectivo en el banco de sangre de un hospital de III nivel en Bogotá, D.C., Colombia. Este banco recolecta sangre de donantes de reposición y de voluntarios dirigidos (quienes donan para un familiar o amigo). Cada donante es entrevistado y sus datos consignados en una encuesta o formulario diseñado por el propio banco de sangre. Se revisaron las encuestas y los resultados serológicos de los donantes que acudieron en noviembre y diciembre de 1996.

Se analizó la utilidad de la encuesta y la selección del donante, teniendo en cuenta los siguientes aspectos:

1) si las preguntas incluian o no factores de riesgo para las diferentes infecciones susceptibles de trasmitirse por sangre;

2) el porcentaje de preguntas con respuesta en todos los casos;

3) la proporción de individuos con y sin factores de riesgo y sus resultados serológicos, y
4) concordancia entre las respuestas del donante y el procedimiento realizado, ya fuera aceptando o no la donación.

Se analizaron, además, las características sociodemográficas de los donantes de sangre. Finalmente, se estimó la reactividad a marcadores serológicos como $\mathrm{VIH}, \mathrm{HBsAg}, \mathrm{VHC}, T$. pallidum y $T$. cruzi.

\section{Resultados}

El banco de sangre en estudio recolectó $2 \%$ del total de unidades de sangre recolectadas en Bogotá durante 1996, con un promedio mensual de 200 unidades. En los meses de estudió se acercaron a donar 414 personas a quienes se les recolectó igual número de unidades de sangre.

La distribución por sexo mostró que $65,9 \%$ correspondió al sexo femenino y el $34,1 \%$ restante al sexo masculino; el rango de edad estuvo entre 18 y 50 años con un promedio de 32 años; las diferencias por sexo y edad no fueron estadísticamente significativas. $50,2 \%$ (208) de los donantes manifestó haber donado sangre alguna vez en su vida y de éstos, $29(14,0 \%)$ lo habían hecho en el último año, aunque no se obtuvo información respecto a si lo habían hecho en este mismo banco de sangre.

Se halló que la encuesta de selección del donante no incluía preguntas sobre antecedentes de tatuajes, acupuntura y uso de drogas intravenosas y sobre el tipo de conducta sexual. Sin embargo, contemplaba preguntas de escasa utilidad y poco específicas como las relativas al hábito de fumar cigarrillo, consumir alcohol y haber estado expuesto a radiografías.

Del total de preguntas contenidas en la encuesta, $90 \%$ fue diligenciado. Las preguntas relativas a la ocupación, el estado civil y el sufrir de asma presentaron el mayor porcentaje sin respuesta.

El $99 \%$ de los donantes no informó sobre antecedentes de ictericia previa, haber estado en contacto con sujetos con hepatitis o haberla padecido, ni antecedentes de haber tomado sangre de armadillo. No se incluyó en el análisis el consumo de alcohol como factor de exclusión debido a que la pregunta no hacia diferencias según grado de consumo. 
De los 414 donantes de sangre, $325(78,5 \%)$ no informaron sobre factor de riesgo alguno para la donación en su encuesta y las unidades de sangre de $52(16 \%)$ de ellos fueron serorreactivas a alguno de los marcadores, mientras que 10 unidades de sangre $(11,23 \%)$ de los 89 donantes que informaron al menos sobre un factor de riesgo fueron serorreactivas, de las cuales, 5 lo fueron a más de un marcador.

Las 62 unidades de sangre serorreactivas a alguno de los marcadores ( $\mathrm{VIH}, \mathrm{HBsAg}, \mathrm{VHC}, T$. pallidum y $T$. cruzi ) corresponden a $15 \%$ del total de sangre donada, siendo la reactividad para el anticuerpo contra $T$. cruzi la más elevada $(4,83 \%)$ (cuadro 1 ).

Los factores de riesgo informados por los 10 donantes reactivos fueron: antecedente de transfusión sanguínea, dos; uno de ellos reactivo a la prueba de Chagas y el otro a la de VHC; antecedentes de radiografía de tórax, cuatro, de los cuales 2 fueron reactivos a $\mathrm{VIH}$, uno a Chagas y uno a VHC; no gozar de buena salud en el momento de la donación, tres, con uno de ellos reactivo para Chagas, otro para $\mathrm{VIH}$ y otro para VHC, y, finalmente, tensión arterial alta en uno de los donantes que fue reactivo a Chagas.

Entre los donantes no reactivos, se hallaron otros factores de riesgo como: antecedentes de uso de drogas estimulantes, haber sufrido paludismo, haber sido vacunado recientemente contra hepatitis, fiebre amarilla o tétanos, haber sido mordido por un perro, haber sufrido enfermedades de transmisión sexual, haber tomado en los últimos 15 días drogas descritas como excluyentes.

Para el análisis de las características sociodemográficas, fueron útiles 228 (55\%)

Cuadro 1. Serorreactividad a los diferentes marcadores en 414 donantes en un banco de sangre de Bogotá, durante noviembre y diciembre de 1996.

\begin{tabular}{lcc}
\hline Marcador & Número & Porcentaje \\
\hline VIH & 13 & 3,14 \\
HBsAg & 5 & 1,21 \\
VHC & 17 & 4,11 \\
Sífilis & 13 & 3,14 \\
Chagas & 20 & 4,83 \\
\hline
\end{tabular}

Fuente: banco de sangre de un hospital de III nivel. encuestas que contenían todas las respuestas correspondientes a sexo, edad, ocupación, estado civil y lugar de residencia, y que se analizaron según el resultado de la reactividad serológica para marcadores como $\mathrm{VIH}, \mathrm{HBsAg}$ y sífilis.

Se observó que las mujeres presentaron un riesgo de 4,13 veces mayor que los hombres de presentar serorreactividad en el tamizaje de estos marcadores $(I C=1,34-12,7)(p<0,01)$.

El mayor riesgo de serorreactividad lo presentaron las mujeres entre 40 y 49 años de edad que están en el hogar y que están casadas o conviven en unión libre. No se presentaron donantes serorreactivos que informaran haber vivido fuera de la ciudad. Las características de ambos grupos se muestran en el cuadro 2.

\section{Discusión}

La falta de inclusión en la encuesta de preguntas sobre antecedentes de tatuajes, acupuntura y uso de drogas IV y sobre tipos de conducta sexual demuestra que ésta no se diseñó teniendo en cuenta la epidemiología de las infecciones a tamizar (agente etiológico, vías de transmisión y sus factores de riesgo), lo cual se fortalece, además, con el hecho de que contemple preguntas poco útiles en la selección del donante como las referentes al consumo de cigarrillo o alcohol y la exposición a radiografías $(1,2)$.

El hecho de que varios donantes $(21,49 \%)$ que informaron, por lo menos, sobre uno de los diferentes factores considerados de exclusión, no fueron excluidos con el fin de proteger tanto al receptor como al donante, podría reflejar desconocimiento del proceso de selección y de su importancia en la seguridad transfusional $(2,7)$. Este hecho se ve reforzado con el hallazgo de varias preguntas sin respuesta, tal como ocurrió con la información sobre las características sociodemográficas, situación que además limita el análisis de las encuestas.

De otro lado, estas prevalencias podrían deberse a un elevado número de resultados falsos positivos en las pruebas de marcadores infecciosos y que no fueron evaluadas por el banco de sangre.

Todo lo anterior, unido a que los donantes de este banco son de reposición o familiares, podría 
Cuadro 2. Características sociodemográficas en donantes de sangre en un banco de sangre de Bogotá durante noviembre y diciembre de 1996 .

\begin{tabular}{|c|c|c|c|c|c|c|c|c|}
\hline \multirow[b]{2}{*}{ Caracteristicas } & \multicolumn{2}{|c|}{$\begin{array}{c}\begin{array}{c}\text { Reactivo a } \\
\text { VIH, HBsAg } \\
\text { anti-T. pallidum }\end{array} \\
n=14\end{array}$} & \multicolumn{2}{|c|}{ 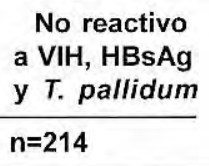 } & \multirow[b]{2}{*}{ Total } & \multirow[b]{2}{*}{$\mathbf{R P}$} & \multirow[b]{2}{*}{ IC } & \multirow[b]{2}{*}{$\mathbf{p}$} \\
\hline & $\mathbf{n}$ & $\%$ & $\mathrm{n}$ & $\%$ & & & & \\
\hline \multicolumn{9}{|l|}{ Edad (años) } \\
\hline $20-29$ & 6 & 6,38 & 88 & 93,61 & 94 & 1,96 & $0,5-7,59$ & 0,49 \\
\hline $30-39$ & 3 & 3,26 & 89 & 96,73 & 92 & 1 & & \\
\hline $40-49$ & 5 & 11,90 & 37 & 88,1 & 42 & 3,65 & $0,91-14,5$ & 0,10 \\
\hline \multicolumn{9}{|l|}{ Sexo } \\
\hline Masculino & 4 & 2,81 & 138 & 97,18 & 142 & 1 & & \\
\hline Femenino & 10 & 11,62 & 76 & 88,37 & 86 & 4,13 & $1,34-12,7$ & $<0,01$ \\
\hline \multicolumn{9}{|l|}{ Ocupación } \\
\hline Empleado & 7 & 4,24 & 158 & 95,75 & 165 & 1,0 & & 1,0 \\
\hline Hogar & 5 & 11,36 & 39 & 88,63 & 44 & 2,69 & $0,89-8,0$ & 0,13 \\
\hline Independiente & 2 & 4,24 & 17 & 89,47 & 19 & 2,48 & $0,55-11,1$ & 0,23 \\
\hline \multicolumn{9}{|l|}{ Estado Civil } \\
\hline Casado & 5 & 5,55 & 85 & 94,44 & 90 & 1,07 & $0,3-3,9$ & 1,0 \\
\hline Unión libre & 4 & 5,12 & 74 & 94,87 & 78 & 1 & & \\
\hline Soltero & 5 & 9,09 & 50 & 90,90 & 55 & 1,77 & $0,5-6,3$ & 0,48 \\
\hline Separados & & & 1 & & 1 & & & \\
\hline Viudos & & & 4 & & 4 & & & \\
\hline \multicolumn{9}{|l|}{ Residencia } \\
\hline En la ciudad & 14 & 7,82 & 165 & 92,17 & 179 & & & \\
\hline Fuera de la ciudad & 0 & & 49 & & 49 & & & \\
\hline
\end{tabular}

Fuente: banco de sangre de un hospital de III nivel.

explicar las altas serorreactividades halladas, con $15 \%$ para todos los marcadores y $3,14 \%$ para anticuerpos contra VIH $(0,3 \%)$, que son 3 y 10 veces superiores a los promedios nacionales informados $(5,2$ y $0,3 \%)$ para todos los bancos en 1996 y a las de otros estudios (3-6).

La elevada prevalencia hallada en el grupo de mujeres de ocupación hogar, casadas o en unión libre y mayores de 40 años puede sugerir una transmisión de la infección a través de su pareja o compañero permanente; por tanto, es necesario desarrollar nuevos estudios que permitan validar o descartar esta hipótesis.

La selección del donante de sangre se hace básicamente a través de los resultados serológicos, hecho que sustenta que $21,49 \%$ de los donantes informó antecedentes o factores de riesgo para la donación y no fueron excluidos y de éstos, $11,23 \%$ fueron posteriormente reactivos a las pruebas serológicas.
La encuesta y el procedimiento seguido en este banco no permiten cumplir el objetivo de seleccionar un donante de bajo riesgo y repetitivo, por lo que sugerimos incluir dentro de la encuesta de selección del donante preguntas que permitan aumentar su capacidad para detectar individuos con factores de riesgo, así como establecer procedimientos claros para la selección de los donantes y capacitar al personal que realiza esta labor sobre la importancia de la encuesta para mejorar la calidad del donante.

Otros factores como la promoción de la donación voluntaria y repetitiva, la monitorización y el análisis de la información de las encuestas y del tamizaje serológico y un control interno de calidad que permita conocer la cantidad de unidades con resultados no esperados como falsos positivos y negativos en las pruebas, permiten evitar situaciones similares a las presentadas en este estudio y aumentan la calidad de la sangre y la seguridad transfusional. 


\section{Agradecimientos}

Los autores expresan sus agradecimientos a Aída Suárez, Carolina Lozada y Nelly Paredes por la información suministrada para el desarrollo de este estudio y a Martha Renza por la traducción del resumen.

\section{Referencias}

1. Glea G. The role of personal interviews by direct questioning on blood donors with particular reference to prevention of transfusion-related infections: a Scottish perspective. Transfusion 1997;7:13-7.

2. Silvergleid AJ, Leparc GF. Impact of explicit questions about high risk activities on donor attitudes and donor deferral patterns: results in two community blood centres. Transfusion 1989;29:362-4.
3. Beltrán M, Ayala M, Ching R. Tamizaje en bancos de sangre. Colombia 1996. Inf Quinc Epidemiol Nac 1997; 2:158-9.

4. World Health Organization, Blood Safety Unit. Developing a national policy and guidelines on the clinical use of blood. Second edition. Geneva: WHO; 1998. p.127.

5. Cortés A, Rojas N P. Marcadores para enfermedades transmitidas por transfusión en diferentes grupos de donantes de sangre. Medicina Transfusional 1994;1:103.

6. Guerrero Romero JF, Castañeda A. Prevalencia y factores de riesgo asociados a hepatitis $C$ en donantes de sangre en el municipio de Durango, México. Salud Pública México 1996;38:94-100.

7. Beltrán M. Malaria transfusional. Inf Quinc Epidemiol Nac 1999;4:279-81. 